\title{
Appendix: Numbers of People who Participated in the Participatory Research
}

\begin{tabular}{|c|c|c|}
\hline Inquiry & Who & Number \\
\hline \multicolumn{3}{|l|}{ Kenya } \\
\hline \multirow[t]{8}{*}{ Mombasa } & Shanzu volunteer involving organisations (VIOs) & 5 \\
\hline & Kongowea $\mathrm{VIOs}$ & 5 \\
\hline & City centre VIOs & 5 \\
\hline & Local research team & 20 \\
\hline & International VSO volunteers & 2 \\
\hline & VIA Network & 10 \\
\hline & Community members spoken to & 400 \\
\hline & Wider Mombasa volunteers & 30 \\
\hline \multirow[t]{3}{*}{ Korogocho } & Involved in participatory sessions & 300 \\
\hline & $\begin{array}{l}\text { Community-based organisations (CBOs) and self-help } \\
\text { organisations }\end{array}$ & 50 \\
\hline & Local volunteers (research team) & 15 \\
\hline \multirow[t]{3}{*}{ Kalifi } & International Citizen Service (ICS) volunteers & 80 \\
\hline & Local volunteers & 30 \\
\hline & Community members & 200 \\
\hline \multirow[t]{4}{*}{ Others } & Volunteer involving organisations network & 20 \\
\hline & International and national volunteers & 50 \\
\hline & Other volunteers & 60 \\
\hline & Total & 1,282 \\
\hline \multicolumn{3}{|l|}{ Mozambique } \\
\hline \multirow[t]{2}{*}{ Aeroporto (Youth church volunteers) } & Youth church volunteers (male and female, 15-28 years) & 10 \\
\hline & Other participants & 200 \\
\hline \multirow[t]{8}{*}{ Kubatsirana (Where's the Bread) } & Director of Kubatsirana & 1 \\
\hline & Manager of Kubatsirana & 1 \\
\hline & Volunteer reps & 2 \\
\hline & Volunteer group 1 (women) & 10 \\
\hline & Volunteer group 2 (20-40 years) & 7 \\
\hline & Pastors & 2 \\
\hline & Activista group (30-50 years) & 12 \\
\hline & Community members & 15 \\
\hline
\end{tabular}


Chamanculo D (Spokes of a Wheel) Male

Female

AEFUM volunteers

Participants

International volunteers

VSO Staff

Boane

Director of Kindlemuka Organisation

Activistas and volunteers (23-60 years)

Participants

AEFUM volunteers

AEFUM volunteer group

Participants 50

Ministry of Youth and Sports 15

UNV representative 100

CNV staff and members 10

Red Cross Mozambique 30

Kutenga

Activistas (17-40 years)

Hospital volunteers and activistas

Community members 70

Formal health volunteers

Local governance representatives

Volunteers

Mothers (supported by volunteers)

Hospital staff

Health committee

Secretario do barrio

Canhandula

Association volunteers

Association Volunteers Board

Nurse

Health post staff

Community members supported by volunteers 5

Beira

Community members

Inhambane

Community members

Total

\section{Nepal}

Education

International volunteers

Teachers

Community members

Students

ECD facilitators 
NDVS

NDVS project officers

Public servants

Key stakeholders in the Nepal volunteering sector

NDVS volunteers

Participants

Community validation events

Stakeholder validation event (INGOs, government officials, NGOs, CBOs)

Total 383

\section{Philippines}

Bohol (generic)

School children/members of youth groups

Residents

Active members of People's Organisations

VSO national volunteer

VSO international volunteers

VSO diaspora volunteer

VSO ICS volunteers (batch 4)

VSO ICS programme supervisors

VSO Bahaginan staff

Host homes

VSO international staff (Asia workshop)

Local government/university staff

Returned ICS volunteers and family

VSO ICS volunteers (batches 5 and 6)

Local volunteers in core research team

Psychology Department (University of the Philippines)

Basdio Youth Group

Adult residents, Basdio

Other youth group leaders

Local government/university staff

Members of People's Organisation and residents

Teachers

Host mother/resident

VSO ICS programme supervisor

VSO Bahaginan staff

TMO local volunteer/student volunteers

Fishermen

Seaweed farmers

Women residents 19

6


Palawan (TMO) (cont.)

Teachers

Out-of-school youth

High school students 28

Elementary students 15

Barangay officials 10

Peripheral actors 8

TMO staff 8

Palawan (Fishermen)

Resident volunteers 15

TMO staff 8

Peripheral actors 8

VSO national volunteer

TMO local volunteers/student volunteers

Barangay officials

VSO Bahaginan staff

Staff members (Jesuit Volunteers Philippines

Foundation, University of Ateneo)

Staff members (Ugnayan ng Pahinungod, UP Los

Bagnos)

Social Work Degree Programme, Bicol University

Fisheries and Aquatic Resources, Bohol Island State

University

Kristohanong Katilingban sa Pagpakabana (KKP), Xavier

University, Cagayan de Oro

Institutional Network for Social Action (UNSA), Miriam

College, Manila

Staff members of the Commission for Higher

Education

National Volunteers Coalition

Three national stakeholder meetings

University of Philippines staff 